\title{
Protótipo de aplicativo móvel motivacional para pessoas com hipertensão arterial sistêmica
}

Prototype of a motivational mobile application for people with hypertension Prototipo de aplicación móvil motivacional para personas con hipertensión arterial sistémica

Taciana da Costa Farias Almeida ${ }^{1}$ ic https://orcid.org/0000-0002-9390-9656

Mailson Marques de Sousa ${ }^{2}$ ib https:/orcid. org/0000-0002-8099-4310

Bernadete de Lourdes André Gouveia ${ }^{3}$ ib https:/orcid.orgy0000-0001-8133-6048

André Atanasio Maranhão Almeida 4 io https://orcid.org/0000-0003-1442-3998

Simone Helena dos Santos Oliveira ${ }^{5}$ io https://orcid.org/0000-0002-9556-1403

Como citar:

Almeida TC, Sousa MM, Gouveia BL, Almeida AA, Oliveira SH. Protótipo de aplicativo móvel motivacional para pessoas com hipertensão arterial sistêmica. Acta Paul Enferm. 2021;34:AAPE001055.

DOI

http://dx.doi.org/10.37689/actaape/2021A0001055

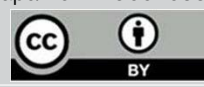

Descritores

Aplicativos móveis; Telefone celular; Hipertensão; Anti-hipertensivos; Autogestão

Keywords

Mobile applications; Cell phone; Hypertension; Anthypertensive agents; Self-management

Descriptores

Aplicaciones móviles; Teléfono celular: Hipertensión; Antihipertensivos; Automanejo

Submetido

5 de Maio de 2020

Aceito

2 de Dezembro de 2020

Autor correspondente

Taciana da Costa Farias Almeida E-mail: tacianacfalmeida@gmail.com

\section{Resumo}

Objetivo: Descrever o processo de produção do protótipo de um aplicativo para smartphone para motivar pessoas com hipertensão arterial sistêmica a aderir ao tratamento medicamentoso.

Métodos: Estudo de produção tecnológica do tipo prototipagem, com construção baseada no modelo design instrucional contextualizado, que seguiu as etapas de análise (levantamento de crenças); design (seleção de ícones, mídias, interface gráfica e validação de conteúdo) e desenvolvimento (construção do protótipo). 0 conteúdo motivacional para composição textual baseou-se nas crenças dos indivíduos com hipertensão arterial sistêmica em observância à Teoria do Comportamento Planejado.

Resultados: 0 protótipo foi composto por vídeo motivacional contendo conceitos, estatísticas e apelos persuasivos relacionados às crenças emitidas; contatos preferenciais; controles de medicamentos com horários e alarmes direcionados, informações sobre os medicamentos em uso, controle de valores da pressão arterial; mensagens persuasivas positivas e negativas; e perfil do usuário.

Conclusão: 0 protótipo é uma inovação tecnológica com potencial para motivar a adesão ao tratamento medicamentoso e diminuição dos níveis pressóricos dos indivíduos com hipertensão.

\section{Abstract}

Objective: To describe the prototype production process for a smartphone application to motivate people with hypertension to adhere to medication treatment.

Methods: This is a study of technological production of the prototyping type, with building based on the contextualized instructional design model, which followed the analysis (survey of beliefs), design (selection of icons, media, graphic interface and content validation), and development (prototype building) steps. The motivational content for textual composition was based on the beliefs of individuals with hypertension in compliance with the Theory of Planned Behavior.

Results: The prototype was composed of motivational video containing concepts, statistics and persuasive appeals related to the beliefs issued; preferred contacts; medication control with targeted schedules and alarms, information about the medications in use; control of blood pressure values; persuasive positive and negative messages; user profile.

Conclusion: The prototype is a technological innovation with the potential to motivate adherence to medication treatment and decrease blood pressure levels in individuals with hypertension. 


\section{Resumen}

Objetivo: Describir el proceso de producción del prototipo de una aplicación para smartphone para motivar a las personas con hipertensión arterial sistémica a adherir al tratamiento medicamentoso.

Métodos: Estudio de producción tecnológica de creación de prototipo, con elaboración basada en el modelo de diseño educativo contextualizado, que consistió en una etapa de análisis (recopilación de creencias), una de diseño (selección de íconos, comunicación, interfaz gráfica y validación de contenido) y una de desarrollo (construcción del prototipo). El contenido motivacional para la composición textual se basó en las creencias de los individuos con hipertensión arterial sistémica en cumplimiento con la teoría del comportamiento planeado.

Resultados: El prototipo está compuesto por un video motivacional que contiene conceptos, estadísticas y llamadas persuasivas relacionadas con las creencias expresadas; contactos preferenciales, control de medicamentos con horarios y alarmas específicas, información sobre los medicamentos en uso, control de valores de la presión arterial; mensajes persuasivos positivos y negativos; y perfil del usuario.

Conclusión: El prototipo es una innovación tecnológica con potencial para motivar la adherencia al tratamiento medicamentoso y la reducción de los niveles de presión de los individuos con hipertensión.

\section{Introdução}

A Hipertensão Arterial Sistêmica (HAS) é considerada um problema de saúde pública, ao se levar em conta uma prevalência de $32 \%$ da população adulta que convive com esta patologia, no contexto brasileiro. ${ }^{(1)}$ É uma das causas mais importantes de mortalidade prematura no mundo, e também um dos fatores de risco mais evitáveis de doenças cardiovasculares, que pode ser facilmente detectada e efetivamente tratada com medicamentos de baixo custo e mudanças no estilo de vida. ${ }^{(1,2)}$

Entre os desafios remanescentes do tratamento medicamentoso da HAS encontram-se a baixa adesão ao tratamento e o descontrole dos níveis de pressão arterial (PA), ${ }^{(3)}$ que reverberam em complicaçóes da doença e em ônus socioeconômico pessoal, familiar e para o setor da saúde. ${ }^{(4)}$ Supóe-se que o aumento da participação do paciente no manejo do comportamento de tomar os comprimidos anti-hipertensivos prescritos seja uma estratégia viável, facilitada por novas tecnologias de intervenção, ${ }^{(5)} \mathrm{e}$ um caminho promissor, ao reduzir o foco nas medidas reativas e estimular medidas proativas, com o propósito de apoiar o autogerenciamento da doença pelo indivíduo, com consequente redução da não adesão. $^{(6)}$

A tecnologia de saúde móvel, frequentemente referida como $m$-health, abrange o uso de smartphones, tablets ou computadores pessoais no gerenciamento de doenças crônicas. ${ }^{(7)}$ É comprovado que estas tecnologias ajudam no autogerenciamento da HAS por meio de definição de alarmes, lembretes para os pacientes tomarem seus medicamentos e en- vio de mensagens motivadoras; ${ }^{(2,4)}$ vinculação de relatórios de aferição da PA dos pacientes ao seu prontuário eletrônico para análise pelos profissionais de saúde; ${ }^{(2,4)}$ fornecimento de feedback aos pacientes sobre sua PA e motivação de comportamentos saudáveis; ${ }^{(4-6)}$ e funcionamento como sensores de PA. ${ }^{(7)}$

Teorias sociais são utilizadas no campo da saúde para compreensão de fenômenos cujo alvo incide em compreender a motivação do indivíduo em executá-los. Destaca-se nesse contexto, a Teoria do Comportamento Planejado (TCP). ${ }^{(8,9)}$ Para TCP a intenção (motivação) é considerada preditora imediata do comportamento e é modulada pela atitude (crenças comportamentais) em relação ao comportamento, normas subjetivas (crenças normativas) e controle comportamental percebido (crenças de controle). ${ }^{(8)}$

Intervenção realizada com base na TCP, que utilizou aplicativo para telefones celulares para enviar mensagens com objetivo de motivar indivíduos com HAS a adotar um estilo de vida saudável, evidenciou modulação favorável ao comportamento revertendo crenças negativas em positivas para o controle dos níveis pressóricos e autogerenciamento da HAS após seis semanas de seguimento. ${ }^{(6)}$ As mensagens desta intervenção foram estruturadas a partir do repertório de crenças e fatores determinantes presentes na população pesquisada, portanto consideraram os aspectos socioculturais e comportamentais do grupo ao qual as mensagens foram dirigidas, o que explica o seu poder de influência segundo a TCP.

Apreende-se que intervençôes de baixo custo, aceitáveis e sustentáveis, para prevenir e tratar efeti- 
vamente a HAS e controle dos níveis pressóricos são essenciais e devem ser o foco de novas tecnologias para melhorar a adesão ao tratamento e, consequentemente, diminuir complicações e a prevalência da doença. ${ }^{(7)}$

No Brasil, destaca-se, nos últimos cinco anos, a construção de aplicativos voltados à gestáo do cuidado em enfermagem, em diferentes âmbitos da assistência e ensino; ${ }^{(10-12)}$ saúde do trabalhador de enfermagem; ${ }^{(13)}$ e acessibilidade direta para o autocuidado com autonomia pelos pacientes. ${ }^{(14,15)}$ Entretanto, nenhum deles aborda aspectos relacionados à HAS e à motivação dos indivíduos para tomar os anti-hipertensivos.

O sistema interativo de suporte e autogerenciamento, proporcionado por tecnologias do tipo celulares e seus recursos visuais concretos, pode favorecer o envolvimento ativo da pessoa com HAS e tem potencial para suportar as transformaçóes atuais dos indivíduos como beneficiários dos cuidados de saúde, além de promover autonomia em relação ao seu próprio bem-estar, relacionado ao tratamento. ${ }^{(2)}$

O sucesso de intervençóes comportamentais para pacientes com condiçôes crônicas geralmente é melhorado, incentivando os pacientes a se envolverem ativamente em seus cuidados. ${ }^{(7)}$ Neste sentido, o telefone celular é o terminal ideal para fornecer serviços de saúde que aprimoram os comportamentos de autogerenciamento dos pacientes na vida diária, uma vez que se trata de um equipamento individual e de suporte pessoal que pode ser acessado sempre que a pessoa necessitar e de acordo com as configuraçôes escolhidas pelo usuário. ${ }^{(6)}$

Diante de tais consideraçóes, mostra-se relevante ao contexto contemporâneo desenvolver novas tecnologias de cuidado que possam auxiliar as estratégias convencionais oferecidas a essa população, com foco em uma autogestão terapêutica eficiente e adequada.

Assim, como proposta inovadora ao cuidado da pessoa com HAS, este estudo objetiva descrever o processo de produção do protótipo de um aplicativo para smartphone para motivar pessoas com hipertensão arterial sistêmica a aderir ao tratamento medicamentoso.

\section{Métodos}

Estudo de desenvolvimento tecnológico ${ }^{(16)}$ do protótipo de um aplicativo (app) que apresenta as funcionalidades para o autogerenciamento da saúde e comunicaçôes e mensagens persuasivas para motivar pessoas com HAS a tomar os comprimidos prescritos para o controle da doença.

Para o desenvolvimento do app optou-se pelo conceito de prototipagem, que consiste na construção de um modelo de software que posteriormente será implantado e avaliado pelo cliente, e então será implementado. ${ }^{(17)}$ Este aconteceu de acordo como método de Design Instrucional Contextualizado (DIC), que é composto de cinco fases: análise, design, desenvolvimento, implementação e avaliação. ${ }^{(18,19)}$

1) Análise: Reconhecida a baixa adesão ao tratamento anti-hipertensivo ${ }^{(3,20)}$ e conforme os preceitos da TCP, que direciona intervençóes para motivar a mudança e/ou fortalecimento de atitudes, normas socias e controle percebido com base nas crenças primárias que guiam a performance do comportamento, foi realizado estudo para analisar crenças dos indivíduos com HAS em relaçáo à tomada dos anti-hipertensivos. ${ }^{(21)}$ Participaram desta etapa de levantamento de crenças 28 pessoas em uso contínuo de anti-hipertensivos, em acompanhamento ambulatorial. Utilizou-se análise de conteúdo para agrupar as crenças (comportamentais, normativas e de controle) mais frequentes na polução em estudo, de modo a compor o jogo modal que subsidiou a elaboração da comunicação audiovisual e mensagens persuasivas.

Na sequência, passou-se à definição dos conteúdos, análise da infraestrutura tecnológica e criação de um diagrama para orientar a construção da ferramenta.

2) Design: Envolveu a definição do conteúdo da comunicação audiovisual persuasiva, a produção das mensagens (com imagens e no formato adequado aos smartphones) com base nas crenças identificadas em estudo prévio; ${ }^{(21)}$ a definição dos ícones; a seleção das mídias e o desenho da interface gráfica (layout). Utilizaram-se os softwares Adobe Photoshop ${ }^{\oplus}$ (prototipagem das telas do app com as mensagens persuasivas e animaçóes das ilustraçóes do vídeo), Adobe Illustrator ${ }^{\circledast}$ (processo de vetorização das ilustraçooes das telas do app e vídeo) e Sony Vegas $\mathrm{PRO}^{\odot}$ 
(montagem, edição e finalização do vídeo). Para estes procedimentos, contou-se com a colaboração de profissional de design especialista em arte e mídia para a elaboração das ilustrações e animaçóes dos recursos audiovisuais.

Após a construção dos recursos audiovisuais, procedeu-se a validação do conteúdo e aparência por um comitê de especialistas. Foram recrutados na Plataforma Lattes uma amostra intencional de 13 especialistas (onze enfermeiros, um fisioterapeuta e um profissional de mídias visuais), que apresentaram no mínimo, um dos seguintes critérios: experiência na área da saúde no atendimento a pacientes com HAS e/ou pesquisas na área de HAS, TCP e Tecnologias da Informação e Comunicação em Saúde.

Cada participante foi esclarecido sobre o objetivo do estudo e recebeu, por meio do google forms, um roteiro e uma escala tipo Likert de quatro pontos: 1 = Discordo Totalmente (não relevante/não representativo/não claro); 2 = Discordo (necessita de revisão para ser representativo/pouco claro); 3 = Concordo (representativo/claro); 4 = Concordo Totalmente (relevante/representativo/muito claro). Após os juízes concluírem o processo de validaçáo foi aplicado o Índice de Validade de Conteúdo (IVC). ${ }^{(22)}$

Os itens avaliados foram apreciados em uma única rodada apresentando IVC $\geq 0,80$. ${ }^{(22)}$ As sugestôes dos especialistas para aprimoramento dos recursos audiovisuais foram analisadas e acatadas e, ao final, o conteúdo da comunicação audiovisual e das mensagens apresentaram-se adequados, claros, pertinentes, abrangentes e com capacidade de persuasão para motivar os indivíduos com HAS a tomar os anti-hipertensivos.

3) Desenvolvimento: Realizado na plataforma Android $^{\circledR}$, com linguagem Java. A escolha desta plataforma se deu pelo acesso fácil a uma biblioteca Java disponibilizada pelo Google. Um dos parâmetros predeterminados foi a utilização de software gratuito, de forma que o custo não interferisse na construção do sistema. ${ }^{(23)}$ Esta etapa compreendeu a construção do protótipo app, com auxílio de analista de sistemas com experiência em programação e desenvolvimento de software, com base nas fases anteriores.

A construção do protótipo (validação de conteúdo e funcionalidades) para implantação nos smartphones de usuários com HAS foi então finalizada. Pesquisa subsequente está sendo desenvolvida envolvendo as fases de implementação e avaliação, cujos resultados seráo divulgados oportunamente.

$\mathrm{O}$ estudo respeitou as normas nacionais e internacionais de pesquisa. O seu desenvolvimento ocorreu entre julho e outubro de 2019, após a aprovação pelo Comitê de Ética em Pesquisa local (CAAE: 79671317.3.0000.5182). Em relação aos direitos autorais das imagens utilizadas, obedeceu-se à Lei no 9.610, de 19 de fevereiro de 1998 .

\section{Resultados}

O app recebeu o nome inicial de "Quali +", abreviação da palavra 'qualidade', em associação ao símbolo matemático '+', que representa a qualidade do tratamento, resultando em mais benefícios e melhorias para o bem-estar.

Foi idealizado um app com ferramentas para lembrar o paciente dos horários das tomadas dos anti-hipertensivos orais prescritos, assim como fornecer mensagens e vídeo motivadores ao tratamento, com informaçôes sobre a doença e os fármacos em uso.

Um vídeo motivacional, construído com base em crenças sobre o tratamento farmacológico, de 3 minutos e 58 segundos, foi inserido em sua tela principal, para que o usuário do app possa conhecer melhor a doença, assim como sentir-se motivado a tomar seus medicamentos a partir das crenças que emanaram do contexto no qual faz parte e que incluíram as vantagens e desvantagens de tomar os comprimidos, as pessoas que apoiam e não apoiam o tratamento e as facilidades e dificuldades para fazê-lo, ${ }^{(21)}$ utilizando-se de linguagem adequada ao público-alvo. A versão final do vídeo produzido pode ser acessada no Youtube no canal do Laboratório de Tecnologias do Cuidar-TecSaúde, link de acesso: https://youtu.be/85aF_JcDqHU.

Associadas ao vídeo, 29 mensagens com conteúdos persuasivos, ilustradas e em formato de tela de app (15 positivas e 14 negativas) também foram inseridas. Estas são apresentadas através de notificação no horário estabelecido pelo paciente e, segundo as orientaçóes médicas, para tomar os fármacos, onde uma delas é selecionada aleatoriamente. A progra- 
mação dos horários pode ser realizada pelo próprio usuário de acordo com a prescrição medicamentosa.

As mensagens foram inseridas para servir como lembretes, motivar o uso do medicamento (positivas), assim como alertar no caso de o comportamento não estar sendo realizado conforme recomendaçóes (negativas). Todas as mensagens poderão ser acessadas pelo usuário no momento em que desejar. A versão final das mensagens, em formato de tela de app também podem ser acessadas no Youtube no canal do Laboratório de Tecnologias do Cuidar - TecSaúde, disponível no link de acesso: https://youtu.be/dTGlCgpC018.

Para ajudar no autogerenciamento da saúde, relacionada a HAS, o app apresenta os recursos: contatos; medicaçóes em uso, que conta com alarmes para os momentos de tomada, gerenciamento de comprimidos (pela contagem e alerta de que é necessário adquirir mais) e registro das últimas aferiçóes da PA; tela inicial com acesso a vídeo persuasivo e visualização do horário da próxima dose

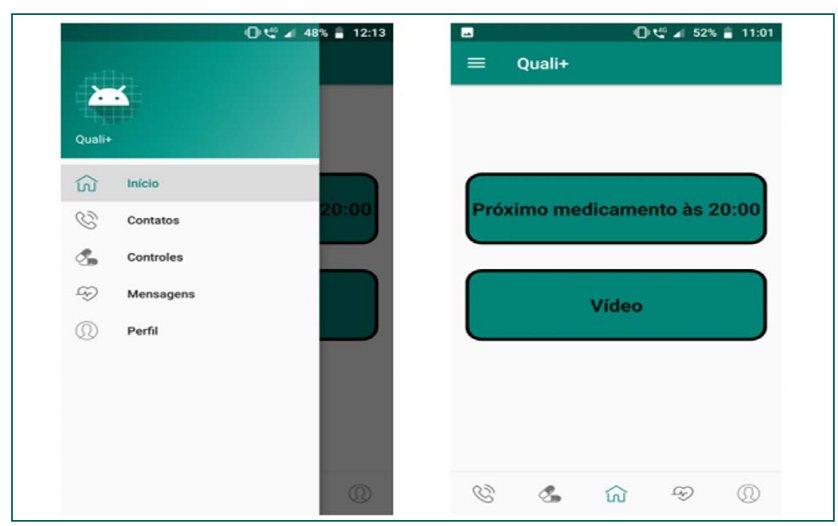

Figura 1. Layouts da tela inicial, acessada por barra de ferramentas e tela cheia do medicamento; mensagens persuasivas positivas e negativas; e informaçóes sobre o perfil do usuário.

A “Tela Inicial” apresenta a informação do próximo horário de tomada do fármaco. Essa programação gera alarme para lembrá-lo de tomar e, ao desligar o alarme, o paciente pode informar ao sistema sobre a sua intenção de realizar a tarefa. Este recurso permite, ainda, acessar o vídeo persuasivo, quando o usuário almejar (Figura 1).

A tela de "Contatos" armazena os números de telefones de emergência, do especialista médico cardiologista ou outro profissional da saúde, como enfermeiro de referência; e cinco pessoas referentes positivas para o indivíduo (pessoas próximas, familiares e/ou amigos), assim como pode ser solicitada ajuda via mensagens com os idealizadores do app.

A tela de "Controles" armazena o nome dos medicamentos em uso para HAS, a contagem desses fármacos, com recurso de aviso quando faltarem dias (programável) para adquirir os medicamentos, informações sobre cada medicamento, programação de horários de tomada com alarme, e visualização das últimas 10 medidas da PA. Salienta-se que essas informaçóes poderão ser inseridas pelo usuário, algum referente social ou profissional de saúde em consonância com a terapêutica prescrita.

Ao clicar sobre o nome do fármaco em uso, é possível obter informaçóes importantes sobre o medicamento, estruturadas em formato de mapa conceitual e linguagem simples, conforme imagem abaixo, que apresenta a tela de 'controles' do aplicativo e um exemplo de informaçôes do fármaco Losartana Potássica 50 mg (Figura 2).

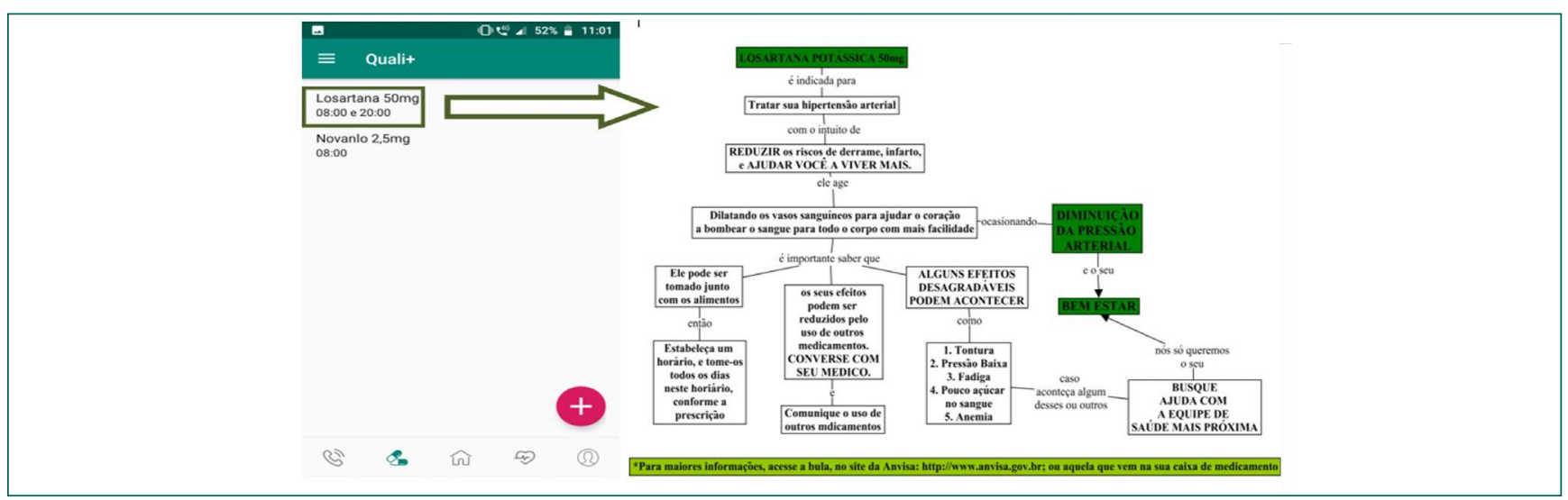

Figura 2. Layout da tela de controles com a informação dos medicamentos em uso, associado a informações sobre o medicamento 


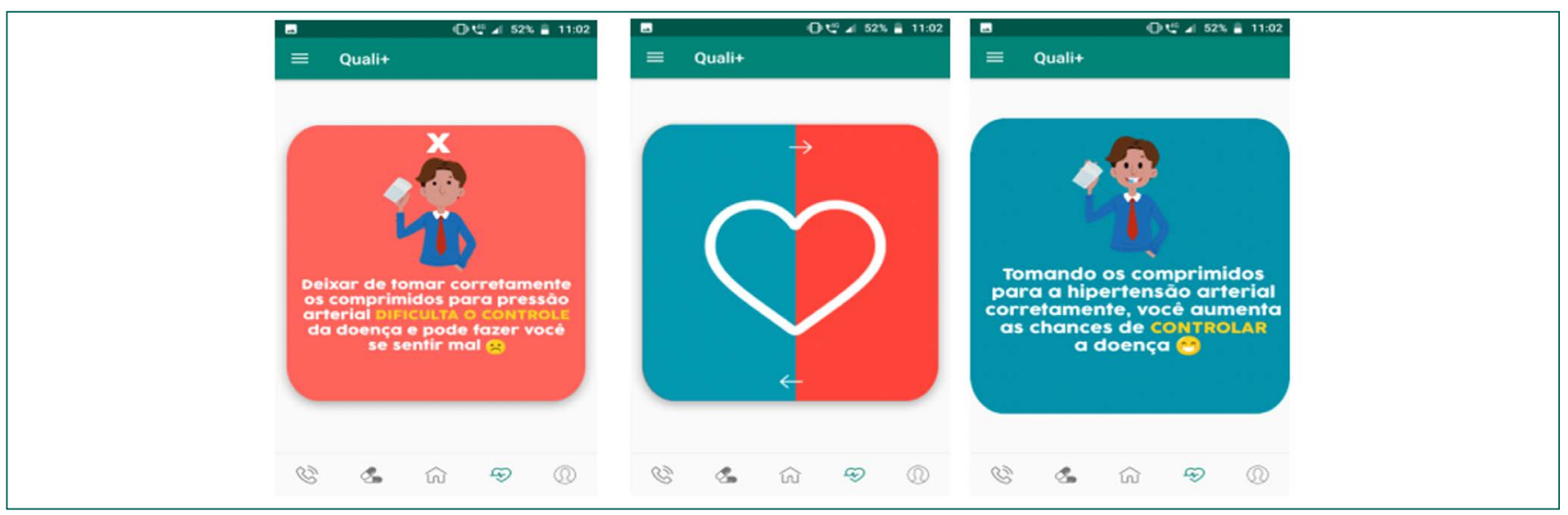

Figura 3. Layouts das telas de mensagem negativa, tela de acesso às mensagens e mensagem positiva

O recurso "Mensagens" armazena as mensagens positivas e negativas com conteúdo motivador e persuasivo, construídas e validadas com base em crenças de indivíduos com HAS. Constituem este recurso 15 mensagens positivas (fundo de tela azul) e 14 mensagens negativas (fundo de tela vermelha). É exibida uma única mensagem por vez e alterna-se para outra, aleatoriamente, ao arrastar a mensagem corrente para a direta (positivas) ou esquerda (negativas) (Figura 3).

No "Perfil" são armazenados os dados pessoais do usuário (foto, nome, data de nascimento, sexo, tipo sanguíneo, cor da pele, estado civil, número de filhos, com quem reside, onde reside, ocupação, escolaridade e alergias medicamentosas).

Direcionadas ao público-alvo deste app, constituído de indivíduos com HAS, atendidos pelo sistema público de saúde, com baixa renda e escolaridade, a facilidade de uso e acesso foram requisitos importantes para esta construção, possibilitando uma integração efetiva entre a ferramenta e o usuário, com seu uso previsível e confortável, a fim de obter o objetivo proposto: ajudar este público em relaçáo ao tratamento medicamentoso anti-hipertensivo.

A projeção deste app se deu para que ele possa ser instalado em dispositivo móvel (smartphone) por meio do Google Play. Neste momento será necessário o acesso à Internet, porém o seu manuseio no cotidiano se dará de forma offline, sem a necessidade de conexão. Optou-se pelo desenvolvimento para a plataforma Android', por ser compatível com a maioria dos smartphones disponíveis no mercado e à população de diferentes classes sociais. Esta é a plataforma de app mobile mais acessível e frequente para o público-alvo.

$\mathrm{O}$ acesso às informaçóes se dará com a manipulação direta dos objetos na tela, por meio do Multi-Touch, que exige que os objetos sejam mais comprometidos com suas tarefas, e facilita a compreensão de suas finalidades, assim como auxilia no controle do app por parte do usuário, pois permite que ele confirme suas intençóes ou cancele açóes antes de concretizá-las (configuraçóes). Apesar da possibilidade de o app sugerir açóes, a decisão deve ser do usuário, para que ele se sinta mais confiante e autoavalie as suas decisóes de cuidado de forma individualizada.

\section{Discussão}

Este é o primeiro aplicativo motivacional construído para ser utilizado em smartphones de pacientes em tratamento anti-hipertensivo no idioma português. As funcionalidades desta tecnologia incluem informações sobre os fármacos em uso, lembretes, controles de medicamentos e valores de pressão arterial, contato com os desenvolvedores, além de outras funcionalidades que implicam em motivação para o autogerenciamento do tratamento medicamentoso para o controle da doença.

A escolha das funçóes do aplicativo considerou outros estudos de desenvolvimento de softwares, ${ }^{(4,7,24)}$ com o intuito de também estimular o comportamento de tomada dos fármacos anti-hipertensivos e controle dos níveis pressóricos. 
Considera-se que este software de autogestão motivacional pode ser uma ferramenta útil na prática clínica para ajudar as pessoas com HAS a autogerenciar a doença, uma vez que, associados ao estímulo para a tomada dos fármacos, a pessoa ainda pode realizar a automedida da PA com equipamento próprio ou na unidade de saúde, registrar estes controles e acompanhar o comportamento dos níveis pressóricos, associado ao uso correto dos fármacos; além de ter um registro de suas ações em relação ao uso dos fármacos, podendo ser repassadas aos profissionais da saúde em momentos de atendimento.

Estudo realizado na Suécia identificou que o uso de aplicativo com objetivo semelhante a este reduziu significativamente os níveis pressóricos elevados dos usuários após oito semanas de implementação, além de considerar que a mudança de foco da adesão à autogestão pode ter sido o motivo do sucesso. ${ }^{(5)}$

Assim, torna-se essencial a implementação desta tecnologia em investigações longitudinais futuras, com o objetivo de avaliar o efeito do aplicativo na motivação de adesão ao comportamento estudado e, como consequência, ponderar sua contribuição na diminuição dos níveis pressóricos por melhoria da adesão ao tratamento.

Identificam-se que intervençôes que têm como propósito motivar o comportamento saudável de aderir ao tratamento visam apenas às crenças pró-adesão, negligenciando processos mais implícitos, como motivos de não adesão. ${ }^{(2,25)}$ Neste sentido, e com o propósito principal de motivar comportamento saudável, optou-se por inserir no aplicativo tanto mensagens positivas quanto negativas, ponderando que os motivos de não adesão precisam ser trabalhados de forma mais clara e compreensiva, para que crenças negativas se transformem em positivas e que motive de fato a realização do tratamento.

Compreende-se que conviver com uma doença crônica como a HAS, pode ocasionar expectativas negativas sobre adoção e mudança no estilo de vida essencial para manutenção da saúde. ${ }^{(26,27)}$ Assim, optou-se por apresentar argumentos persuasivos negativos com o intuito de provocar uma avaliação das consequências do náo uso dos medicamentos anti-hipertensivos. Ressalta-se que a abordagem empregada náo teve o intuito de punição, mas de alertar para possíveis complicaçóes decorrentes da baixa ou não adesão à terapêutica prescrita e melhorar o desempenho do comportamento. Essa abordagem corrobora com estudo que empregou mensagens positivas e negativas na intenção de realizar o autocuidado em pessoas com diabetes, obtendo êxito com a negativa. ${ }^{(28)}$

Acredita-se que a comunicação persuasiva inserida no formato audiovisual, mensagens positivas e negativas, além de informaçóes sobre a doença, os fármacos em uso, oferecem reflexão e oportunidade para as pessoas com HAS compreenderem suas condiçóes de saúde, assim como promover mudanças no comportamento de tomada dos fármacos. ${ }^{(4,9)}$ A utilização do aplicativo poderá proporcionar um tratamento proativo, autonomia, segurança e autoconfiança para autogerenciar a saúde de acordo com as configurações que o usuário escolher. $\mathrm{O}$ acesso ao conteúdo de maneira atrativa, com recursos de imagens e sons, facilidade para programação do regime terapêutico permitirá ao usuário ampliar o conhecimento acerca do tratamento, e incentivo ao comportamento saudável de tomar os anti-hipertensivos conforme prescrito.

É importante salientar que na tecla controles, existe a flexibilidade para edição de novas informaçóes sobre a terapêutica medicamentosa prescrita, horários de uso e registros das aferições da PA, que pode ser realizada pelo próprio usuário. Para atenuar possíveis vieses de baixa escolaridade, a configuração inicial pode ser feita pelo profissional de saúde nas consultas para o manejo clínico da HAS. No futuro, outros avanços podem ser incorporados para potencializar os registros e melhor atender as especificidades dos usuários, na medida que novas versôes forem sendo testadas e demandas forem surgindo durante o uso.

Apesar de a baixa adesão ao tratamento anti-hipertensivo ser um problema mundial e ter-se conhecimento da existência de outros aplicativos disponíveis na literatura internacional, ${ }^{(2,4,6,7)}$ a aplicação daqueles no cenário e contexto local torna-se inviável, uma vez que o idioma e a adaptação cultural não se adequariam à realidade vivenciada, já que é um produto tecnológico elaborado para a realidade contextual. Assim, buscou-se construir um app no 
idioma português e que se assemelhasse àqueles em relação a algumas funções que obtiveram sucesso no tratamento naquele contexto, com o objetivo de atender às crenças locais deste público-alvo.

A ausência de tecnologias deste tipo no cenário brasileiro resulta em dificuldades de comparaçóes de desenvolvimentos contextuais, sendo este estudo pioneiro na área de enfermagem e cuidado a pessoas com hipertensão arterial por meio do autogerenciamento da HAS, com base nas crenças sobre o tratamento e construção de um aplicativo para celulares que proporcione autonomia aos usuários assistidos.

Cabe salientar que protótipos de aplicativos recentemente desenvolvidos no Brasil que se voltavam aos usuários do sistema de saúde focavam especificamente a educaçáo de pessoas com doenças arterial periférica $^{(15)}$ e orientações educativas de procedimentos perioperatório ortognático. ${ }^{(14)}$ Estes sistemas também foram desenvolvidos com base no modelo $\mathrm{DIC}^{(18,19)}$ e apresentavam o propósito de tornar conhecidos para estes públicos a doença e os cuidados pós-operatórios, náo incluído o autogerenciamento de uma doença crônica com comportamentos específicos, como no protótipo desenvolvido.

Como limitaçóes deste estudo, aponta-se a ausência das etapas de implementação e avaliação do protótipo. Devido aos aspectos relacionados ao tempo dispendido nas etapas preliminares para construção (validação de conteúdo da comunicação audiovisual e mensagens) e aos custos financeiros, não foi possível dar seguimento imediato às etapas subsequentes. Salienta-se que este estudo deve ser aperfeiçoado e analisado por especialistas em sistemas em relação a sua interface e que pesquisa subsequente será conduzida a fim de prosseguir com as etapas de validação de funcionabilidade e usabilidade do protótipo pelo público-alvo.

Espera-se que, após as fases de implementação e avaliação, o app possa ser utilizado na prática clínica como estratégia adjuvante ao cuidado apoiado à terapêutica prescrita da HAS. Aos enfermeiros, a ferramenta representa uma inovação de particular importância para a consulta de enfermagem, já que as imagens e animações presentes na comunicação persuasiva conferem maior clareza às informaçóes sobre a doença, como o medicamento age e ainda fortalece e esclarece as crenças que buscam motivar e ou desmotivar o uso dos medicamentos.

Ao longo do tratamento, os usuários podem recorrer ao aplicativo para rever comunicaçóes e mensagens, bem como ajustá-lo de acordo com as mudanças no tratamento, de modo a fomentar a autogestáo do uso de fármacos, a partir de um conteúdo qualificado, atrativo, de fácil utilização e compreensível para o público-alvo.

\section{Conclusão}

O protótipo do aplicativo construído para motivar pessoas com hipertensão arterial sistêmica é um recurso tecnológico inovador que permite ao indivíduo em uso de comprimidos anti-hipertensivos: acesso a mensagens e vídeo com conteúdos validados de cunho motivador; mais conhecimento sobre a doença e os fármacos em uso; alarmes com lembretes do horário de tomada dos fármacos; controles e cuidados individualizados.

\section{Colaborações}

Almeida TCF, Sousa MM, Gouveia BLA, Almeida AAM e Oliveira SHS colaboraram com a concepção do estudo, análise e interpretação dos dados, redação do artigo, revisão crítica relevante do conteúdo intelectual e aprovação da versão final a ser publicada.

\section{Referências}

1. Malachias M, Plavnik FL, Machado CA, Malta D, Scala LC, Fuchs S. 7th Brazilian Guideline of Arterial Hypertension: Chapter 1 - Concept, Epidemiology and Primary Prevention. Arq Bras Cardiol. 2016;107(3 Suppl 3):1-6.

2. Bengtsson U, Kjellgren K, Hallberg I, Lundin M, Mäkitalo Å. Patient contributions during primary care consultations for hypertension after self-reporting via a mobile phone self-management support system. Scand J Prim Health Care. 2018;36(1):70-9.

3. Campos CL, Pierin AM, Pinho NA. Hypertension in patients admitted to clinical units at university hospital: post-discharge evaluation rated by telephone. einstein (Sao Paulo). 2017;15(1):45-9.

4. Thangada ND, Garg N, Pandey A, Kumar N. The emerging role of mobile-health applications in the management of hypertension. Curr Cardiol Rep. 2018;20(9):78. 
5. Bengtsson U, Kjellgren K, Hallberg I, Lindwall M, Taft C. Improved Blood Pressure Control Using an Interactive Mobile Phone Support System. J Clin Hypertens (Greenwich). 2016;18(2):101-8.

6. Sun N, Rau PLP, Li Y, Owen T, Thimbebly H. Design and evaluation of a mobile phone-based health intervention for patients with hypertensive condition. Comput Hum Behav. 2016; 63: 98e105.

7. Chen MJ, Chen KY, Chiang SJ, Daimon M, Lee JS, Yu EW, et al. A telehealth service model for the treatment of hypertension. J Telemed Telecare. 2013;19(5):238-41.

8. Ajzen I. The theory of planned behavior. Organ Behav Hum Decis Process. 1991;50(2):179-211.

9. Fishbein M, Aizen I. Predicting and changing behavior: the reasoned action approach. New York: Routledge; 2015. 518p.

10. Silva CP, Dell'Acqua MC, Corrente JE, Castro MC, Kornoff DC. Application development for pressure ulcer indicator. J Health Inform. 2016;8(4):134-41.

11. Pereira IM, Bonfim D, Pirs HH, Góes RF, Gaidzinski RR. Mobile application for data collection in health research. Acta Paul Enferm. 2017;30(5):479-88.

12. Vêscovi SJ, Primo CC, Sant'Anna HC, Bringuete ME, Rohr RV, Prado TN, et al. Mobile application for evaluation of feet in people with diabetes mellitus. Acta Paul Enferm. 2017;30(6):607-13.

13. Gama LN, Tavares CM. Desenvolvimento e avaliação de aplicativo móvel na prevenção de riscos osteomusculares no trabalho de enfermagem. Texto Contexto Enferm. 2019;28:e20180214.

14. Sousa CS, Turrini RN. Development of an educational mobile application for patients submitted to orthognathic surgery. Rev Lat Am Enfermagem. 2019;27:e3143.

15. Mendez CB, Salum NC, Junkes C, Amante LN, Mendez CM. Mobile educational follow-up application for patients with peripheral arterial disease. Rev Lat Am Enfermagem. 2019;27:e3122.

16. Lacerda MR, Costenaro RG. Metodologias da pesquisa para a enfermagem e saúde: da teoria à prática. Porto Alegre: Moriá; 2015. $511 p$.
17. Sperandio DJ, Évora YD. Planejamento da assistência de enfermagem: proposta de um software-protótipo. Rev Lat Am Enfermagem. 2005;13(6):937-43

18. Parreira Júnior WM, Pradela IP, Oliveira LN. 0 uso da norma 14598 na avaliação de software com relação à qualidade. Intercursos. 2009;8(1):63-72.

19. Barra DC, Paim SM, Sasso GT, Colla GW. Métodos para desenvolvimento de aplicativos móveis em saúde: revisão integrativa da literatura. Texto Contexto Enferm. 2017;26(4):e2260017.

20. Rêgo AD, Radovanovic CA. Adherence of hypertension patients in the Brazil's Family Health Strategy. Rev Bras Enferm. 2018;71(3):1030-7.

21. Almeida TC, Sousa MM, Pessoa MS, Sousa LS, Gouveia BL, Oliveira $\mathrm{SH}$. Beliefs of individuals with systemic arterial hypertension related to drug treatment. Rev Rene. 2019;20:e41585.

22. Coluci MZ, Alexandre NM, Milani D. [Construction of measurement instruments in the area of health]. Cien Saude Colet. 2015;20(3):925-36.

23. Grossi LM, Pisa IT, Marin HF. Oncoaudit: development and evaluation of an application for nurse auditors. Acta Paul Enferm. 2014;27(2):179-85.

24. Bengtsson U, Kasperowski D, Ring L, Kjellgren K. Developing an interactive mobile phone self-report system for self-management of hypertension. Part 1: patient and professional perspectives. Blood Press. 2014;23(5):288-95.

25. Herrera PA, Moncada L, Defey D. Understanding Non-Adherence From the Inside: Hypertensive Patients' Motivations for Adhering and Not Adhering. Qual Health Res. 2017;27(7):1023-34

26. Sousa MM, Gouveia BLA, Almeida TFC, Freire MLM, Oliveira SHS. Beliefs of people with salt-related heart failure. Rev Enferm UERJ (Rio de Janeiro). 2019;(27): e44197.

27. Gouveia BL, Sousa MM, Almeida TD, Sousa VA, Oliveira SH. Beliefs related to insulin use in people with Type 2 Diabetes Mellitus. Rev Bras Enferm. 2020 Apr;73(3):e20190029.

28. Park J, Kim SH, Kim JG. Effects of message framing and health literacy on intention to perform diabetes self-care: A randomized controlled trial. Diabetes Res Clin Pract. $2020 ; 161: 108043$. 